EPJ Web of Conferences 61, 04008 (2013)

DOI: $10.1051 /$ epjconf/ 20136104008

(C) Owned by the authors, published by EDP Sciences, 2013

\title{
Probing the Radio Counterpart of Gamma-ray Flaring Region in 3C 84
}

\author{
Hiroshi Nagai ${ }^{1, a}$, Monica Orienti ${ }^{2,3}$, Motoki Kino ${ }^{3}$, Akihiro Doi ${ }^{6}$, Gabriele Giovanniniं ${ }^{2,3}$, Keiichi Asada ${ }^{4}$, Filippo \\ D'Ammando' ${ }^{2}$, Takafumi Haga ${ }^{5}$, Marcello Giroletti ${ }^{2}$, and Kazuhiro Hada ${ }^{2,1}$ \\ ${ }^{1}$ National Astronomical Observatory of Japan, Osawa 2-21-1, Mitaka, Tokyo 181-8588, Japan \\ ${ }^{2}$ INAF Istituto di Radioastronomia, via Gobetti 101, 40129, Bologna, Italy \\ ${ }^{3}$ Dipartimento di Astronomia, Universita' di Bologna, via Ranzani 1, I-40127, Bologna, Italy \\ ${ }^{4}$ Institute of Space and Astronautical Science, Japan Aerospace Exploration Agency, Yoshinodai 3-1-1, Chuo-ku, Sagamihara, \\ Kanagawa 229-8510, Japan \\ ${ }^{5}$ Institute of Astronomy and Astrophysics, Academia Sinica. P.O. Box 23-141, Taipei 10617, Taiwan, R.O.C. \\ ${ }^{6}$ The Graduate University for Advanced Studies, Yoshinodai 3-1-1, Chuo-ku, Sagamihara, \\ Kanagawa 229-8510, Japan
}

\begin{abstract}
The radio source 3C 84 associated with the radio/giant elliptical galaxy NGC 1275 is one of the best targets to probe the radio counterpart of the $\gamma$-ray emitting region. Although this source shows clear time variability in $\gamma$-ray bands, no clear correlation in radio light curve was found on the timescale of $\gamma$-ray variability. The location of the $\gamma$-ray flaring region has been an open question. In this proceeding, we firstly review our previous findings from radio observations. Next we present our new results based on the Very Long Baseline Array (VLBA) data at $43 \mathrm{GHz}$. We discover the limb-brightened structure in the "restarted" jet associated with the 2005 radio outburst. In 1990s, the jet structure was rather ridge-brightening than limbbrightening, despite the observations were done with similar angular resolution. This indicates that the radio jet morphology in terms of the transverse structure has been indeed changed recently. This change in the morphology shows an interesting agreement with the time variation of the $\gamma$-ray flux density, i.e., the $\gamma$-ray flux density in 1990s was more than 7 times lower than the current one. We argue the possibility that the transition from ridge-brightening to limb-brightening is related to the $\gamma$-ray time variability.
\end{abstract}

\section{Introduction}

The radio source $3 \mathrm{C} 84$ is associated with the giant elliptical/radio galaxy NGC 1275 ( $\mathrm{z}=0.0176)$. Thanks to its brightness and proximity, this source is one of the best studied radio source in history. Recently increased activity starting in 2005 was detected in radio band [1]. The VLBI observations revealed that this flux density increase originated within the central pc-scale core, accompanying the ejection of a new jet component [13, hereafter Paper I]. This new component appeared from the south of the core around 2003 [17, hereafter Paper II], and was moving to the position angle $\sim 160^{\circ}$ steadily with slightly changing speed in both parallel and perpendicular directions. The apparent speed was ranging from $0.1 c$ to $0.5 c$. The flux density of both the new component and the core particularly became bright after 2007-2008. After 2009, the new component has still shown monotonic increase in flux density [14, hereafter Paper III] .

It is notable that $3 \mathrm{C} 84$ is the best studied $\gamma$-ray radio galaxy along with M 87 and Centaurus A, and therefore these sources the ideal laboratories to study the $\gamma$-ray emission mechanism in misaligned Active Galactic Nuclei

\footnotetext{
ae-mail: 190 hiroshi.nagai@nao.ac.jp
}

(AGNs). Strong variability in the $\gamma$-ray emission has been detected from 3C 84 by Fermi-LAT. An averaged $\gamma$-ray flux during the first four months is $(2.10 \pm 0.23) \times 10^{-7} \mathrm{ph}$ $\mathrm{cm}^{-2} \mathrm{~s}^{-1}$ above $100 \mathrm{MeV}$ [1]. This $\gamma$-ray flux is seven times brighter than the upper limit by EGRET/CGRO. It was claimed that the innermost jet of $3 \mathrm{C} 84$ is the most likely source of $\gamma$-ray emission because of the time variation on the timescales of years to decades. During the first 2 years, two episodes of increased $\gamma$-ray activity were observed: one occurred in 2009 Apr-May [10], and the other one occurred in 2010 June-August [4]. In particular, the second flare was detected up to $102.5 \mathrm{GeV}$ [2]. Largest increased activity in $\mathrm{GeV}$ band was detected more recently.

\section{Our Previous Findings}

\subsection{No radio counterpart of $\gamma$-ray flares}

In Paper III, the radio time variability was studied to search the correlation with the $\gamma$-ray variability, but no clear correlation was found on the timescale of $\gamma$-ray variability. Neither new component ejection nor change in morphology associated with the $\gamma$-ray flares were found by Very Long Baseline Interferometry (VLBI) observations (Paper III). The lack of significant changes in radio band for 


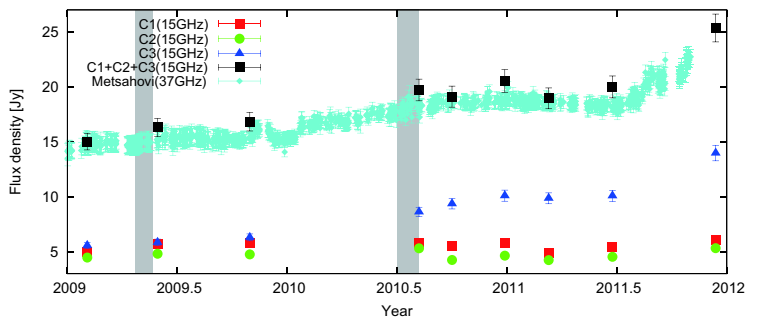

Figure 1. Single dish and VLBI light curves from Metsahovi at $37 \mathrm{GHz}$ and MOJAVE at $15 \mathrm{GHz}$, respecctively. Red squares, green circles, and blue triangles represent the MOJAVE flux densities of $\mathrm{C} 1, \mathrm{C} 2$, and C3, respectively (see Paper II for the component labeling). The sum of flux densities of $\mathrm{C} 1, \mathrm{C} 2$, and $\mathrm{C} 3$ is indicated by black squares. Single dish light curve by Metsähovi is indicated by cyan diamonds. Two $\gamma$-ray flaring events are indicated by pink gray rectangles. Note that Metsähovi flux density contains the emission from the entire 3C 84 source.

3C 84 after the detection of high $\gamma$-ray activity leaves the debate on the region responsible for the high-energy emission and its location is still open.

\subsection{SED modeling using measured apparent motion}

Previous spectral modeling using one-zone synchrotronself Compton (SSC) requires mildly relativistic jet flow in order to reproduce the Fermi $\gamma$-ray flux density [1]. On the other hand, the apparent speed detected by VLBI is relatively slower than the jet speed predicted from onezone SSC model unless the jet angle to the line of sight is very small $\left(<5^{\circ}\right.$ : Paper II). We performed SED fit to the observed broad band spectrum using VLBI-measured apparent speed (Figure 2). If we adopt $\gamma_{\text {min }}=10^{3}$ as is the case in Abdo et al. (2009), the $43 \mathrm{GHz}$ lies below the frequency of low-energy cutoff and therefore the spectral index should be $1 / 3$. However, the observed spectral index of newly-born component (C3) is about -0.9 between $22 \mathrm{GHz}$ and $43 \mathrm{GHz}$. It failed to reproduce the optically thin radio spectrum observed by the Very Long Baseline Array (VLBA) observations (Paper II). Hence it seems difficult to attribute the broadband SED to the one-zone SSC emission from $\mathrm{C} 3$.

\section{Where is the radio counterpart of the $\gamma$-ray flares?}

While several possibilities to reconcile the discrepancy between radio and $\gamma$-ray properties have been discussed (Paper I; Paper II; Paper III), it is of great interest to investigate the presence of spine-sheath structure with the velocity gradient [6] in the jet of 3C 84 since this scenario is one of the successful scenarios to reconcile the $\gamma$-ray emission from the radio galaxies. According to this scenario, the radio emission is mostly coming from the slower sheath while the emission from the spine is beaming away from the line of sight. Therefore, the limb-brightening can be observed along the jet if the spine-sheath structure is

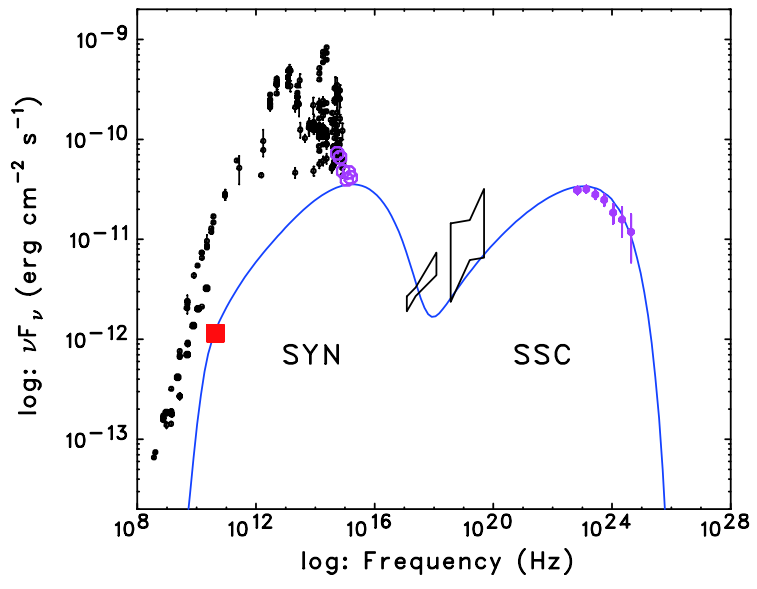

Figure 2. Overall SED of NGC 1275 constructed with multiband data and the VLBA $43 \mathrm{GHz}$ flux of C3 (red square). The SED is fitted to the VLBA $43 \mathrm{GHz}$ flux of C3 (red square), optical/UV (Swift/UVOT; magenta open circle), and $\gamma$-ray (Fermi; magenta point) data with a one-zone SSC model. See Paper II in more detail.

present. Clear evidence of limb-brightening was found in several AGNs [7-9, 15], but no clear signature of limbbrightening has been found in $3 \mathrm{C} 84$. To detect the limbbrightening, high resolution which can resolve the transverse direction of the jet and high dynamic range image are required.

\section{New 43-GHz Very Long Baseline Array Observation}

The observation was carried out with 10 VLBA stations at $43 \mathrm{GHz}$ on 2013 January 24. Both right-hand and left-hand circular polarizations were received, and only parallel-hand correlations (RR and LL) were processed. The observation consisted of many different scans for $3 \mathrm{C} 84$. The integration time for each scan is ten seconds. Total integration time for $3 \mathrm{C} 84$ is about $45 \mathrm{~min}-$ utes, and overall observation including calibrator scans were spanned over 8 hours. The scans for 3C 84 were spread over different hour angle evenly, and therefore we obtained good $u v$-coverage.

The data reduction was performed using Astronomical Imaging Processing System (AIPS) developed by National Radio Astronomy Observatory (NRAO). An a priori amplitude calibration was performed using aperture efficiency and system noise temperature provide by each station. The opacity correction for the atmospheric attenuation was applied. Fringe fitting and bandpass calibration were applied. For the deconvolution of synthesized image, we used CLEAN and self-calibration technique. Final images were obtained after a number of iterations with CLEAN and both phase and amplitude self-calibration using the DIFMAP software package 


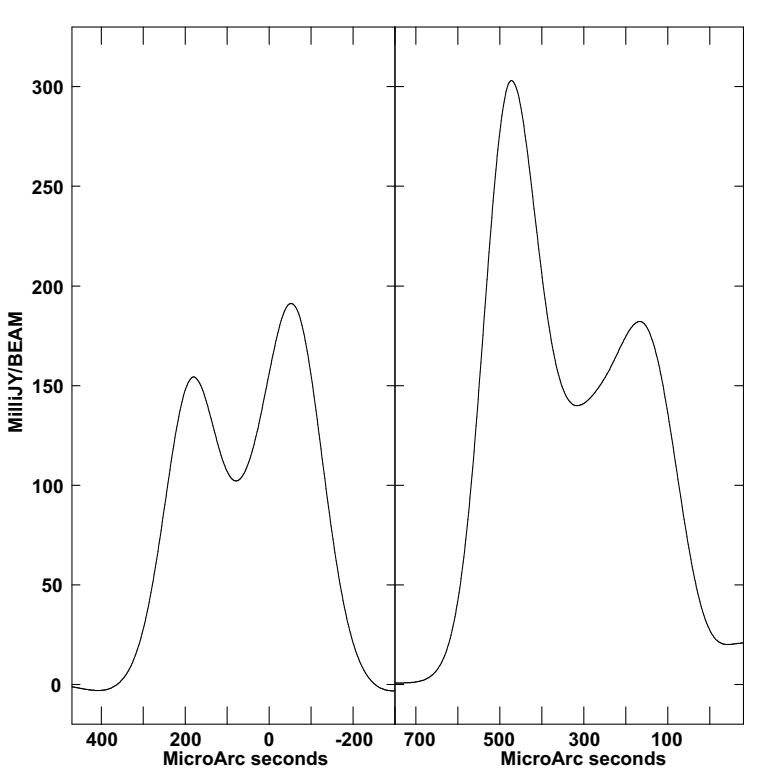

Figure 4. The transverse slice profile of the jet. The left and right figures show the profiles along (a) and (b) indicated by the broken lines in the Fig. 3, respectively.

\section{The $43-\mathrm{GHz}$ VLBA Image of $3 \mathrm{C} 84$}

Figure 3 shows the self-calibrated image of 3C 84 by Very Long Baseline Array observation at $43 \mathrm{GHz}$ on 2013 January 24 . In previous studies it was shown that the source mostly consisted of three components. We have also detected same structure, but finer scale structure is visible from this image. The bright core and one-sided jet structure is clearly seen. The jet is directing to the position angle $-170^{\circ}$ up to 1.2 mas from the core and then slightly changing its direction to the position angle $-180^{\circ}$. At the end of the jet, there is a bright knot-like feature. While this feature was represented by a single Gaussian component, so-called C3, in the previous studies, multiple subcomponents are seen from this image. The changing pattern of the jet direction is approximately consistent with the previously detected path of C3 motion (Paper II). There is an elongated feature $(\mathrm{C} 2)$ towards the west from $\mathrm{C} 3$ region, which invokes the backflow from C3. However, C2 had been already present before the emergence of C3. Therefore, the origin of $\mathrm{C} 2$ is not very clear. We did not detect any significant emission from the counter-jet side. Overall structure is consistent with the images shown by Boston University Blazar Peoject.

The slice profiles across the jet are shown in Figure 4. The most remarkable finding is that the limb-brightened structure is evident along the approaching jet, which is the "restarted" jet associated with an ongoing activity started from 2005. To our knowledge this is the first discovery of the limb-brightening in the jet of $3 \mathrm{C} 84$.

\section{Discussion}

The detection of limb-brightening is expected as if there is the velocity gradient across the jet and the beaming-cone angle of the emission from the "spine" is smaller than the jet viewing angle. So far we have not detected a clear correlation in light curve between radio and $\gamma$-ray in 3C 84 on the timescale of days to months. Also, the observed apparent motion is relatively slower than the jet velocities expected from the one-zone synchrotron-self Compton model or decelerating jet model. Yet, such an uncorrelation between radio and $\gamma$-ray is expected as if we are only seeing the slower sheath by radio observation and $\gamma$-ray emissions are produced by the inverse Compton scattering off the low energy photons from the sheath or spine by the electrons in the fast spine.

Here we roughly estimate the outflow velocity of the limb-brightened region. For the simplicity, we assume that the jet has intrinsically uniform brightness across the jet in transverse direction and the observed transverse brightness is only affected by the transverse velocity structure which results in the varying Doppler enhancement of different jet axisymmetric layers. The limb-brightened structure is corresponding to a layer where the Doppler factor becomes its maximum. Assuming the viewing angle $(\theta)$ of $25^{\circ}$, the Doppler factor reaches its maximum where the bulk Lorentz factor $(\Gamma)$ is about $2.4(\beta \sim 0.9)$. Thus, one can estimate that the velocity of the limb-brightened region (sheath) and the inner dim region (spine) are $\Gamma \sim 2.4$ and $\Gamma \gtrsim 2.4$, respectively. Although the assumed viewing angle is within the range of preferred viewing angle estimated previously

The estimated bulk Lorentz factor of limb-brightening region is somewhat faster than the VLBI measured motion of C3 unless the viewing angle is very small. In [10], it is noted that both the low- and high-energy peaks of NGC 1275's spectral energy distribution (SED) in the $v F_{v}$ representations are located at substantially lower frequencies than those of typical low-frequency peaked BL Lac objects (LBL). Therefore, the jet viewing angle of 3C 84 is not likely to be as small as those of blazars. A possible explanation is that $\mathrm{C} 3$ is the terminal shock produced by the interaction with the interstellar medium (ISM) and apparent motion of $\mathrm{C} 3$ does not reflect the jet motion.

[5], [16], and [12] reported the 43-GHz VLBA images of 3C 84 as of 1990s. From those images, no clear limbbrightening was confirmed despite similar angular resolution was achieved. One possibility to explain this difference is a change in the transverse velocity structure in the epoch $2000-2005$, confirmed by the restarted activity of 3C 84 in 2005 and by the emission of a new bright component (C3) in 2003 (Paper II). This can be realized if the flow speed in spine region was slower in 1990s and the beaming angle of the emission from the spine was larger than the viewing angle. Alternatively, one may think that there was a similar transverse velocity structure in 1990s but the viewing angle was rather small. If the jet viewing angle was smaller than the beaming angle of the spine emission, the ridge-brightening could appear only in 1990s because of strong beaming of the spine emission towards the observer. A change in the viewing angle is suggested by the change of the direction in the plane of 


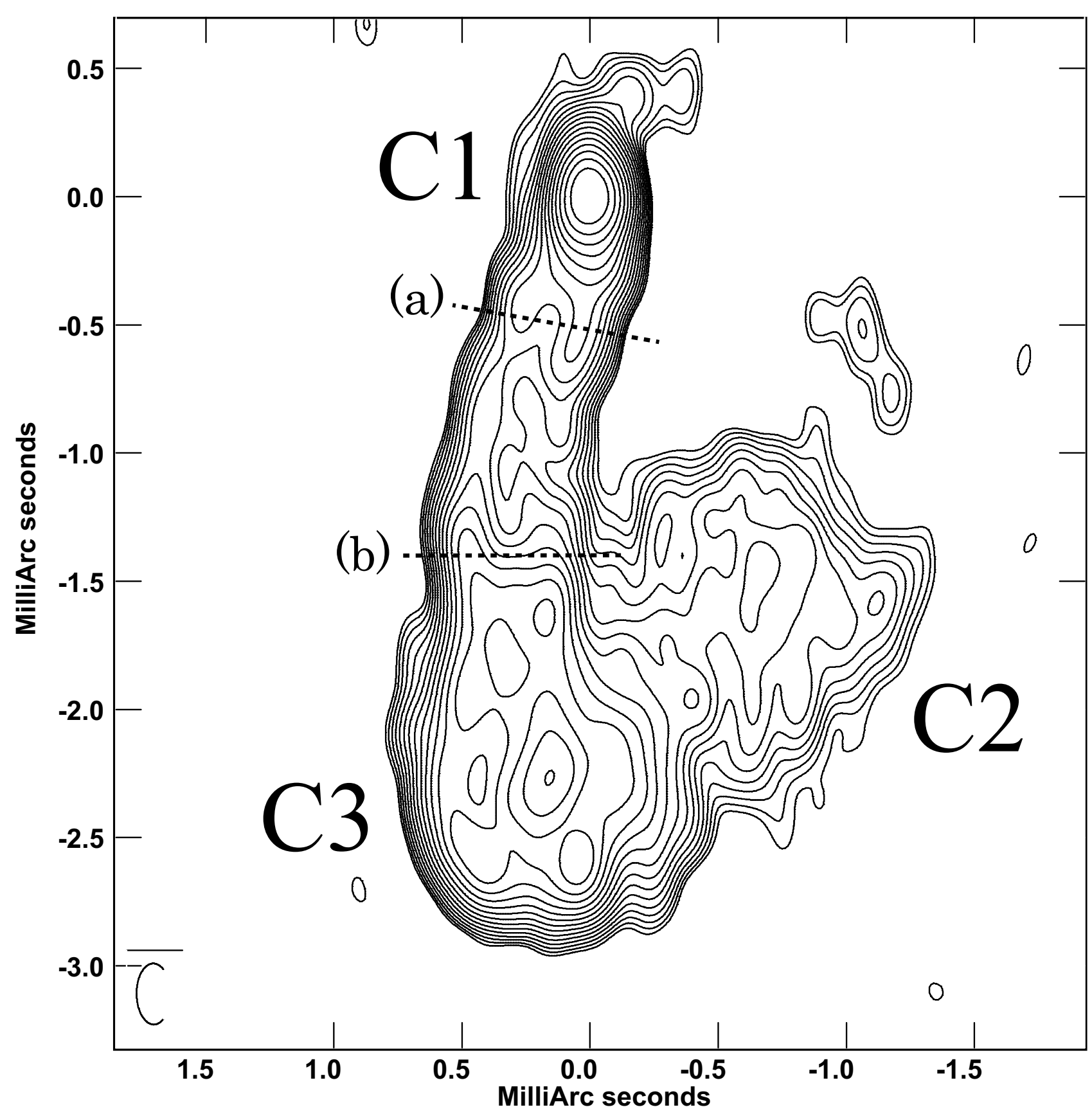

Figure 3. The $43-\mathrm{GHz}$ total intensity map of $3 \mathrm{C} 84$. The contours are plotted at the level of $5.43 \times(-1.41,1,1.41,2.83,4,5.66$, $8,11.3,16,22.6,32,45.3,64,90.5,128,181,256)$ mJy beam $^{-1}$. The peak intensity is $2.17 \mathrm{Jy} \mathrm{beam}^{-1}$. The ellipse shown at the bottom left corner of the image indicates the full-width half-maximum of the convolved beam. The FWHM of the convolved beam is $0.24 \times 0.13$ mas at the position angle $0.69^{\circ}$. The white broken lines (a) and (b) indicated in the figure is the slice locations for Fig. 4 .

the sky of the present jet $(\mathrm{C} 3)$ with respect to pre-existing component C2 (Paper I; Paper II).

We favor the first possibility for the following reason. If the viewing angle was small and the physical properties of the jet (such as magnetic field, electron energy distribution, and etc) were no so different from the current ones, 3C 84 should be detected as the bright $\gamma$-ray source because of the strong beaming effect. Contrary to this, the upper limit of $\gamma$-ray flux in 1990s was more than 7times weaker than the recent flux level. Therefore, we speculate that the change in the apparent transverse struc- ture is resulted from the change in the velocity structure. We propose that the origin of $\gamma$-ray flux variability on the timescale of decades in $3 \mathrm{C} 84$ might be the result of the change in the transverse velocity structure, i.e., the velocity of the spine has became faster than that of 1990s.

\section{Summary and Conclusions}

The new 43-GHz VLBA observation revealed the clear limb-brightened structure along the restarted jet of 3C 84 in the central sub-parsec scale. This is the first discovery 
of the limb-brightening in $3 \mathrm{C} 84$. Interestingly, the limbbrightening was not found in 1990s despite the similar angular resolution was already achieved. This may indicate that the transverse velocity structure was changed in association with the restarted activity in the period of 20002005. If we relate the change of the apparent jet structure with the change in the transverse velocity structure, the time variability of the $\gamma$-ray emission can be naturally explained.

\section{References}

[1] Abdo, A. A., Ackermann, M., Ajello, M., et al. 2009, ApJ, 699, 31

[2] Aleksić, J., Alvarez, E. A., Antonelli, L. A., et al. 2012, A\&A, 539, L2

[3] Asada, K., Kameno, S., Shen, Z.-Q., et al. 2006, PASJ, 58, 261

[4] Brown, A. M., \& Adams, J. 2011, MNRAS, 413, 2785

[5] Dhawan, V., Kellermann, K. I., \& Romney, J. D. 1998, ApJL, 498, L111

[6] Ghisellini, G., Tavecchio, F., \& Chiaberge, M. 2005, A\&A, 432, 401
[7] Giroletti, M., Giovannini, G., Cotton, W. D., et al. 2008, A\&A, 488, 905

[8] Giovannini, G., Taylor, G. B., Arbizzani, E., et al. 1999, ApJ, 522, 101

[9] Junor, W., Biretta, J. A., \& Livio, M. 1999, Nature, 401,891

[10] Kataoka, J., Stawarz, Ł., Cheung, C. C., et al. 2010, ApJ, 715, 554

[11] Lister, M. L., Cohen, M. H., Homan, D. C., et al. 2009, AJ, 138, 1874

[12] Lister, M. L. 2001, ApJ, 562, 208

[13] Nagai, H., Suzuki, K., Asada, K., et al. 2010, PASJ, 62, L11 (Paper I)

[14] Nagai, H., Orienti, M., Kino, M., et al. 2012, MNRAS, 423, L122 (Paper III)

[15] Piner, B. G., Pant, N., \& Edwards, P. G. 2010, ApJ, 723,1150

[16] Romney, J. D., Benson, J. M., Dhawan, V., et al. 1995, Proceedings of the National Academy of Science, 92,11360

[17] Suzuki, K., Nagai, H., Kino, M., et al. 2012, ApJ, 746, 140 (Paper II)

[18] Walker, R. C., Dhawan, V., Romney, J. D., Kellermann, K. I., \& Vermeulen, R. C. 2000, ApJ, 530, 233 